\title{
A PEROV TYPE THEOREM FOR CYCLIC CONTRACTIONS AND APPLICATIONS TO SYSTEMS OF INTEGRAL EQUATIONS
}

\author{
ADRIAN MAGDAŞ
}

Received 12 April, 2016

\begin{abstract}
In this paper we will prove a fixed point theorem of Perov type for cyclic contractions on complete generalized metric spaces. Then, as an application, we will study the existence, uniqueness and approximation of the solution for a system of integral equations.
\end{abstract}

2010 Mathematics Subject Classification: 47H10; 45G15

Keywords: generalized metric space, fixed point, cyclic representation, matrix convergent to zero, cyclic $S$-contraction, system of integral equations, successive approximation

\section{Preliminaries}

We begin the considerations with some notions and results which will be useful further in this paper.

Let $(X, d)$ be a metric space. We denote:

$$
\begin{aligned}
P(X) & :=\{Y \subset X \mid Y \text { is nonempty }\} ; \\
P_{c l}(X) & :=\{Y \in P(X) \mid Y \text { is closed }\} ;
\end{aligned}
$$

If $T: Y \subseteq X \rightarrow X$ is a single-valued operator, then the symbol

$$
\mathrm{F}_{\mathrm{T}}:=\{\mathrm{x} \in \mathrm{Y} \mid \mathrm{x} \in \mathrm{Tx}\}
$$

denotes the fixed point set of $T$.

Definition 1. A matrix $S \in \mathcal{M}_{p}\left(\mathbb{R}_{+}\right)$is called a matrix convergent to zero if $S^{k} \rightarrow 0$ as $k \rightarrow+\infty$.

Theorem 1 ([5], [4]). Let $S \in \mathcal{M}_{p}\left(\mathbb{R}_{+}\right)$. The following statements are equivalent:

(i) $S$ is a matrix convergent to zero;

(ii) $S^{k} x \rightarrow 0$ as $k \rightarrow+\infty, \forall x \in \mathbb{R}^{p}$;

(iii) $I_{p}-S$ is non-singular and

$$
\left(I_{p}-S\right)^{-1}=I_{p}+S+S^{2}+\ldots
$$

(iv) $I_{p}-S$ is non-singular and $\left(I_{p}-S\right)^{-1}$ has nonnegative elements;

(v) $\lambda \in \mathbb{C}, \operatorname{det}\left(S-\lambda I_{p}\right)=0$ imply $|\lambda|<1$. 
The matrices convergent to zero were used by A.I. Perov [2] to generalize the contraction principle in the case of metric spaces with a vector-valued distance.

Definition 2 ([5]). Let $(X, d)$ be a metric space with $d: X \times X \rightarrow \mathbb{R}_{+}^{p}$ a vectorvalued distance and $T: X \rightarrow X$. The operator $T$ is called an $S$-contraction if there exists a matrix $S \in \mathcal{M}_{p}\left(\mathbb{R}_{+}\right)$such that:

(i) $S$ is a matrix convergent to zero;

(ii) $d(T(x), T(y)) \leq S d(x, y), \forall x, y \in X$.

Theorem 2 (Perov, [2]). Let $(X, d)$ be a complete metric space with $d: X \times X \rightarrow$ $\mathbb{R}_{+}^{p}$ a vector-valued distance and $T: X \rightarrow X$ be an $S$-contraction. Then:

(i) $T$ has a unique fixed point $x^{*} \in X$;

(ii) $T^{k} x \stackrel{d}{\longrightarrow} x^{*}$ as $k \rightarrow+\infty$, for all $x \in X$;

(iii) $d\left(T^{k} x, x^{*}\right) \leq S^{k}\left(I_{p}-S\right)^{-1} d(x, T x)$, for all $x \in X$ and $k \in \mathbb{N}$;

(iv) $d\left(x, x^{*}\right) \leq\left(I_{p}-S\right)^{-1} d(x, T x)$ for all $x \in X$.

Another consistent generalization of the contraction principle was given by Kirk, Srinivasan and Veeramani, using the concept of cyclic operator.

Theorem 3 ([1]). Let $\left\{A_{i}\right\}_{i=1}^{m}$ be nonempty subsets of a complete metric space, and suppose $T: \bigcup_{i=1}^{m} A_{i} \rightarrow \bigcup_{i=1}^{m} A_{i}$ satisfies the following conditions (where $\left.A_{m+1}=A_{1}\right)$ :

(1) $T A_{i} \subseteq A_{i+1}$ for $1 \leq i \leq m$;

(2) $\exists k \in(0,1)$ such that $d(T x, T y) \leq k d(x, y), \forall x \in A_{i}, y \in A_{i+1}$, for $1 \leq$ $i \leq m$.

Then $T$ has a unique fixed point.

This theorem suggested the introduction of the following

Definition 3 ([3]). Let $X$ be a nonempty set, $m$ a positive integer and $T: X \rightarrow X$ an operator. By definition, $\bigcup_{i=1}^{m} A_{i}$ is a cyclic representation of $X$ with respect to $T$ if:

(i) $X=\bigcup_{i=1}^{m} A_{i}$, with $A_{i} \in P(X)$, for $1 \leq i \leq m$;

(ii) $T A_{i} \subseteq A_{i+1}$, for $1 \leq i \leq m$, where $A_{m+1}=A_{1}$.

\section{MAIN RESULTS}

Definition 4. Let $(X, d)$ be a metric space with $d: X \times X \rightarrow \mathbb{R}_{+}^{p}$ a vector-valued distance, $A_{1}, \ldots, A_{m} \in P_{c l}(X)$ and $T: X \rightarrow X$ be an operator. If: 
(i) $\bigcup_{i=1}^{m} A_{i}$ is a cyclic representation of $X$ with respect to $T$;

(ii) there exists a matrix $S \in \mathcal{M}_{p}\left(\mathbb{R}_{+}\right)$convergent to zero such that $d(T x, T y) \leq S \cdot d(x, y)$, for any $x \in A_{i}, y \in A_{i+1}$, where $A_{m+1}=A_{1}$, then, by definition, we say that $T$ is a cyclic $S$-contraction.

Theorem 4. Let $(X, d)$ be a complete metric space with $d: X \times X \rightarrow \mathbb{R}_{+}^{p}$ a vectorvalued distance, $A_{1}, A_{2}, \ldots, A_{m} \in P_{c l}(X)$. If $T: X \rightarrow X$ is a cyclic $S$-contraction then the following statements hold:

(1) $T$ has a unique fixed point $x^{*} \in \bigcap_{i=1}^{m} A_{i}$ and the Picard iteration $\left\{x_{n}\right\}_{n \geq 0}$ given by

$$
x_{n}=T x_{n-1}, n \geq 1,
$$

converges to $x^{*}$ for any starting point $x_{0} \in X$;

(2) the following estimates hold:

$$
\begin{aligned}
& d\left(x_{n}, x^{*}\right) \leq S^{n}\left(I_{p}-S\right)^{-1} d\left(x_{0}, x_{1}\right), n \geq 1 ; \\
& d\left(x_{n}, x^{*}\right) \leq\left(I_{p}-S\right)^{-1} d\left(x_{n}, x_{n+1}\right), n \geq 1 ;
\end{aligned}
$$

(3) for any $x \in X$,

$$
d\left(x, x^{*}\right) \leq\left(I_{p}-S\right)^{-1} d(x, T x) .
$$

Proof. (1)

$$
\begin{aligned}
d\left(x_{n}, x_{n+1}\right) & =d\left(T x_{n-1}, T x_{n}\right) \leq S d\left(x_{n-1}, x_{n}\right) \\
& \leq \ldots \leq S^{n} d\left(x_{0}, x_{1}\right)
\end{aligned}
$$

For $k \geq 1$ we have

$$
\begin{aligned}
d\left(x_{n}, x_{n+k}\right) & \leq S^{n} d\left(x_{0}, x_{1}\right)+S^{n+1} d\left(x_{0}, x_{1}\right)+\ldots+S^{n+k-1} d\left(x_{0}, x_{1}\right) \\
& =S^{n}\left(I_{p}+S+S^{2}+\ldots+S^{k-1}\right) d\left(x_{0}, x_{1}\right) \\
& \leq S^{n}\left(I_{p}+S+S^{2}+\ldots\right) d\left(x_{0}, x_{1}\right) \rightarrow 0 \text { as } n \rightarrow \infty,
\end{aligned}
$$

which means that $\left(x_{n}\right)_{n \geq 0}$ is a Cauchy sequence.

$(X, d)$ is a complete metric space, so the sequence $\left(x_{n}\right)_{n \geq 0}$ is convergent to a $q \in X$.

The sequence $\left(x_{n}\right)_{n \geq 0}$ has an infinite number of terms in each $A_{i}, i=\overline{1, m}$, so from each $A_{i}$ one we can extract a subsequence of $\left(x_{n}\right)_{n \geq 0}$ which converges to $q=$ $\lim _{n \rightarrow \infty} x_{n}$.

Because $A_{i}$ are closed, $q \in \bigcap_{i=1}^{m} A_{i}$, so $\bigcap_{i=1}^{m} A_{i} \neq \varnothing$. 
Let be the restriction $\left.T\right|_{i=1} ^{m} A_{i}: \bigcap_{i=1}^{m} A_{i} \rightarrow \bigcap_{i=1}^{m} A_{i}$.

$\bigcap_{i=1}^{m} A_{i}$ is also complete. Applying Perov's theorem, $\left.T\right|_{i=1} ^{m} A_{i}$ has a unique fixed point, which can be obtained by means of the Picard iteration starting from any initial point. It remains to prove that the Picard iteration converges to $x^{*}$, for any initial guess $x \in X$.

$$
\begin{aligned}
d\left(x_{n+1}, x^{*}\right) & =d\left(T x_{n}, T x^{*}\right) \leq S d\left(x_{n}, x^{*}\right) \\
& \leq \ldots \leq S^{n} d\left(x_{0}, x^{*}\right) \rightarrow 0 \text { as } n \rightarrow \infty .
\end{aligned}
$$

(2) $d\left(x_{n}, x_{n+k}\right) \leq d\left(x_{n}, x_{n+1}\right)+d\left(x_{n+1}, x_{n+2}\right)+\ldots+d\left(x_{n+k-1}, x_{n+k}\right)$

$$
\begin{aligned}
& \leq d\left(x_{n}, x_{n+1}\right)+S d\left(x_{n}, x_{n+1}\right)+\ldots+S^{k-1} d\left(x_{n}, x_{n+1}\right) \\
& =\left(I_{p}+S+\ldots+S^{k-1}\right) d\left(x_{n}, x_{n+1}\right), \text { for any } n \in \mathbb{N}, k \geq 1 .
\end{aligned}
$$

Using the statement (iii) from Theorem 1, by letting $k \rightarrow \infty$ in (2.4) and (2.5) we obtain the estimates (2.1) and (2.2).

(3) Let $x \in X$. For $n=0, x_{0}:=x$, the a posteriori estimate (2.2) becomes

$$
d\left(x, x^{*}\right) \leq\left(I_{p}-S\right)^{-1} d(x, T x) .
$$

Theorem 5. (Data dependence theorem) Let $T: X \rightarrow X$ be as in Theorem 4 with $F_{T}=\left\{x_{T}^{*}\right\}$. Let $U: X \rightarrow X$ be an operator such that:

(i) $U$ has at least one fixed point $x_{U}^{*}$;

(ii) there exists $\eta>0$ such that

$$
d(T x, U x) \leq \eta, \text { for any } x \in X .
$$

Then $d\left(x_{T}^{*}, x_{U}^{*}\right) \leq \eta\left(I_{p}-S\right)^{-1}$.

Proof. By letting $x:=x_{U}^{*}$ in the inequality (2.3), we have

$$
\begin{aligned}
d\left(x_{U}^{*}, x_{T}^{*}\right) & \leq\left(I_{p}-S\right)^{-1} d\left(x_{U}^{*}, T x_{U}^{*}\right)=\left(I_{p}-S\right)^{-1} d\left(U x_{U}^{*}, T x_{U}^{*}\right) \\
& \leq\left(I_{p}-S\right)^{-1} \eta .
\end{aligned}
$$

Theorem 6. Let $T: X \rightarrow X$ be as in Theorem 4. Then the fixed point problem for $T$ is well posed, that is, assuming there exist $z_{n} \in X, n \in \mathbb{N}$ such that $d\left(z_{n}, T z_{n}\right) \rightarrow$ 0 , as $n \rightarrow \infty$, this implies that $z_{n} \rightarrow x^{*}$, as $n \rightarrow \infty$, where $F_{T}=\left\{x^{*}\right\}$. 
Proof. By letting $x:=z_{n}$ in the inequality (2.3), we have

$$
d\left(z_{n}, x^{*}\right) \leq\left(I_{p}-S\right)^{-1} d\left(z_{n}, T z_{n}\right), n \in \mathbb{N}
$$

and letting $n \rightarrow \infty$ we obtain $d\left(z_{n}, x^{*}\right) \rightarrow 0, n \rightarrow \infty$.

\section{AN APPLICATION TO A SYSTEM OF INTEGRAL EQUATIONS}

We apply the results given by Theorem 2.1 to study the existence and the uniqueness of solutions of the following system of integral equations:

$$
\left\{\begin{array}{l}
x_{1}(t)=\int_{a}^{b} G_{1}(t, s) f_{1}\left(s, x_{1}(s), x_{2}(s)\right) d s \\
x_{2}(t)=\int_{a}^{b} G_{2}(t, s) f_{2}\left(s, x_{1}(s), x_{2}(s)\right) d s
\end{array}, t \in[a, b]\right.
$$

where $a, b \in \mathbb{R}, a<b$,

$$
\begin{gathered}
G_{1}, G_{2} \in C([a, b] \times[a, b],[0, \infty)), \\
f_{1}, f_{2} \in C([a, b] \times \mathbb{R} \times \mathbb{R}, \mathbb{R}) .
\end{gathered}
$$

Theorem 7. We suppose that:

(i) there exist $\alpha_{k}, \beta_{k} \in C([a, b], \mathbb{R}), m_{k}, M_{k} \in \mathbb{R}$ with $m_{k} \leq \alpha_{k}(t) \leq \beta_{k}(t) \leq$ $M_{k}$, for any $t \in[a, b]$, such that

$$
\left\{\begin{array}{l}
\alpha_{k}(t) \leq \int_{a}^{b} G_{k}(t, s) f_{k}\left(s, \beta_{1}(s), \beta_{2}(s)\right) d s \\
\beta_{k}(t) \geq \int_{a}^{b} G_{k}(t, s) f_{k}\left(s, \alpha_{1}(s), \alpha_{2}(s)\right) d s
\end{array} \text { for } k \in\{1,2\}\right.
$$

(ii) there exist $a_{1}, b_{1}, a_{2}, b_{2} \in \mathbb{R}_{+}$such that

$$
\begin{aligned}
& \left|f_{1}\left(s, u_{1}, u_{2}\right)-f_{1}\left(s, v_{1}, v_{2}\right)\right| \leq a_{1}\left|u_{1}-v_{1}\right|+a_{2}\left|u_{2}-v_{2}\right|, \\
& \left|f_{2}\left(s, u_{1}, u_{2}\right)-f_{2}\left(s, v_{1}, v_{2}\right)\right| \leq b_{1}\left|u_{1}-v_{1}\right|+b_{2}\left|u_{2}-v_{2}\right|,
\end{aligned}
$$

for any $s \in[a, b]$ and $u_{k}, v_{k} \in \mathbb{R}$, with

$$
\left\{\begin{array} { l } 
{ u _ { k } \leq M _ { k } } \\
{ v _ { k } \geq m _ { k } }
\end{array} \quad \text { or } \quad \left\{\begin{array}{l}
u_{k} \geq m_{k} \\
v_{k} \leq M_{k}
\end{array} \text { for } k \in\{1,2\} ;\right.\right.
$$

(iii) $\sup _{t \in[a, b]} \int_{a}^{b} G_{k}(t, s) d s \leq 1$ for $k \in\{1,2\}$;

(iv) $f_{k}$ is decreasing in each of the last two variables, that is, $u_{1}, u_{2}, v_{1}, v_{2} \in \mathbb{R}, u_{1} \leq v_{1}, u_{2} \leq v_{2} \Rightarrow f_{k}(s, u, v) \geq f_{k}\left(s, u_{2}, v_{2}\right)$, for any $s \in[a, b]$, and $k \in\{1,2\}$; 
(v) the matrix $S=\left(\begin{array}{ll}a_{1} & a_{2} \\ b_{1} & b_{2}\end{array}\right)$ converges to zero.

Then the system (3.1) has a unique solution $x^{*}=\left(x_{1}^{*}, x_{2}^{*}\right) \in C\left([a, b], \mathbb{R}^{2}\right)$, with $\alpha_{k} \leq$ $x_{k}^{*} \leq \beta_{k}$, for $k \in\{1,2\}$.

This solution can be obtained by the successive approximations method, starting at any element $x^{0} \in C\left([a, b], \mathbb{R}^{2}\right)$. Moreover, if $x^{n}$ is the $n^{\text {th }}$ successive approximation, then we have the following estimation:

$$
\left\|x^{*}-x^{n}\right\| \leq S^{n}\left(I_{2}-S\right)^{-1}\left\|x^{0}-x^{1}\right\|,
$$

where

$$
\|x\|=\left(\begin{array}{c}
\left|x_{1}\right|_{\infty} \\
\left|x_{2}\right|_{\infty}
\end{array}\right) \quad \text { and }|x|_{\infty}=\max _{t \in[a, b]}|x(t)| .
$$

Proof. Let us denote

$$
\begin{gathered}
X:=\left(C([a, b], \mathbb{R}),|\cdot|_{\infty}\right), Z=X \times X \\
\|\cdot\|: Z \rightarrow \mathbb{R}^{2},\|x\|=\left\|\left(x_{1}, x_{2}\right)\right\|=\left(\begin{array}{c}
\left|x_{1}\right|_{\infty} \\
\left|x_{2}\right|_{\infty}
\end{array}\right),
\end{gathered}
$$

where $\left|x_{k}\right|_{\infty}=\max _{t \in[a, b]}\left|x_{k}(t)\right|$ is the Chebyshev norm.

Then $(Z,\|\cdot\|)$ is a generalized Banach space.

We consider the following closed subsets of $X$ :

$$
\begin{aligned}
& A_{1}=\left\{\left(x_{1}, x_{2}\right) \in Z \mid x_{k} \leq \beta_{k}, k \in\{1,2\}\right\}, \\
& A_{2}=\left\{\left(x_{1}, x_{2}\right) \in Z \mid x_{k} \geq \alpha_{k}, k \in\{1,2\}\right\},
\end{aligned}
$$

and the operator $T: Z \rightarrow Z$,

$$
\begin{gathered}
\left(x_{1}, x_{2}\right)=x \mapsto T x=\left(T_{1} x, T_{2} x\right), \\
T_{k} x(t):=\int_{a}^{b} G_{k}(t, s) f_{k}\left(s, x_{1}(s), x_{2}(s)\right) d s, \text { for } k \in\{1,2\} .
\end{gathered}
$$

The system (3.1) is equivalent with the equation $T x=x$.

We will prove that $A_{1} \cup A_{2}$ is a cyclic representation of $Z$ with respect to $T$. Let $x=\left(x_{1}, x_{2}\right) \in A_{1} \Rightarrow x_{k}(s) \leq \beta_{k}(s), \forall s \in[a, b]$, for $k \in\{1,2\}$.

Using the monotonicity of $f_{k}$ we have

$$
G_{k}(t, s) f_{k}\left(s, x_{1}(s), x_{2}(s)\right) \geq G_{k}(t, s) f_{k}\left(s, \beta_{1}(s), \beta_{2}(s)\right), \text { for } k \in\{1,2\}
$$

and from (i), by integration,

$$
\int_{a}^{b} G_{k}(t, s) f_{k}\left(s, x_{1}(s), x_{2}(s)\right) d s \geq \alpha_{k}(t)
$$

which means that

$$
T_{k} x(t) \geq \alpha_{k}(t), \forall t \in[a, b], \text { for } k \in\{1,2\} \Rightarrow T x \in A_{2} .
$$


So $T A_{1} \subseteq A_{2}$. In a similar way we have $T A_{2} \subseteq A_{1}$.

Using the conditions (ii) and (iii) we have

$$
\begin{aligned}
\left|T_{k} x(t)-T_{k} y(t)\right| & \leq \int_{a}^{b} G_{k}(t, s)\left|f_{k}\left(s, x_{1}(s), x_{2}(s)\right)-f_{k}\left(s, y_{1}(s), y_{2}(s)\right)\right| d s \\
& \leq \int_{a}^{b} G_{k}(t, s)\left(a_{k}\left|x_{1}(s)-y_{1}(s)\right|+b_{k}\left|x_{2}(s)-y_{2}(s)\right|\right) d s \\
& \leq \int_{a}^{b} G_{k}(t, s)\left(a_{k}\left|x_{1}-y_{1}\right|_{\infty}+b_{k}\left|x_{2}-y_{2}\right|_{\infty}\right) \\
& \leq a_{k}\left|x_{1}-y_{1}\right|_{\infty}+b_{k}\left|x_{2}-y_{2}\right|_{\infty}, \forall t \in[a, b] \\
& \Rightarrow\left|T_{k} x-T_{k} y\right|_{\infty} \leq a_{k}\left|x_{1}-y_{1}\right|_{\infty}+b_{k}\left|x_{2}-y_{2}\right|_{\infty} \\
& \Rightarrow\left(\begin{array}{c}
\left|T_{1} x-T_{1} y\right|_{\infty} \\
\left|T_{2} x-T_{2} y\right|_{\infty}
\end{array}\right) \leq S\left(\begin{array}{c}
\left|x_{1}-y_{1}\right|_{\infty} \\
\left|x_{2}-y_{2}\right|_{\infty}
\end{array}\right),
\end{aligned}
$$

so we have

$$
\|T x-T y\| \leq S\|x-y\| \text {, for any }(x, y) \in A_{1} \times A_{2},
$$

and by the condition (v) it results that the operator $T$ is a cyclic $S$-contraction.

All the conditions of Theorem 4 are satisfied, so $T$ has a unique fixed point

$$
x^{*}=\left(x_{1}^{*}, x_{2}^{*}\right) \in A_{1} \cap A_{2}, \text { with } \alpha_{k} \leq x_{k}^{*} \leq \beta_{k}, \text { for } k \in\{1,2\} .
$$

This finishes the proof.

Further on, we will study the continuous dependence phenomenon for the system (3.1).

We consider the perturbed system of integral equations

$$
\left\{\begin{array}{l}
y_{1}(t)=\int_{a}^{b} H_{1}(t, s) g_{1}\left(s, y_{1}(s), y_{2}(s)\right) d s \\
y_{2}(t)=\int_{a}^{b} H_{2}(t, s) g_{2}\left(s, y_{1}(s), y_{2}(s)\right) d s
\end{array}\right.
$$

where

$$
H_{1}, H_{2} \in C([a, b] \times[a, b],[0, \infty)), \quad g_{1}, g_{2} \in C([a, b] \times \mathbb{R} \times \mathbb{R}, \mathbb{R}) .
$$

Theorem 8. We suppose that the conditions of Theorem 7 are satisfied and we denote by $x^{*}$ the unique solution of the system of integral equations (3.1).

If $y^{*} \in C\left([a, b], \mathbb{R}^{2}\right)$ is a solution of the perturbed system of integral equations (3.6), and

$$
\sup _{t \in[a, b]} \int_{a}^{b} H_{k}(t, s) d s \leq 1,
$$


then we have the following estimation:

$$
\left\|x^{*}-y^{*}\right\|_{\mathbb{R}^{2}} \leq\left(I_{2}-S\right)^{-1}(\eta+\tau),
$$

where $\eta=\left(\eta_{1}, \eta_{2}\right), \tau=\left(\tau_{1}, \tau_{2}\right)$ and

$$
\left\{\begin{array}{l}
\eta_{k}=\sup \left\{\left|f_{k}(s, u, v)\right| \mid s \in[a, b], u, v \in \mathbb{R}\right\}, \\
\tau_{k}=\sup \left\{\left|g_{k}(s, u, v)\right| \mid s \in[a, b], u, v \in \mathbb{R}\right\},
\end{array} \text { for } k \in\{1,2\} .\right.
$$

Proof. We consider the operator $T: Z \rightarrow Z$ attached to the system (3.1), defined by the relation (3.5).

Let $U: Z \rightarrow Z$ be an operator attached to the perturbed system (3.6) and defined by the relation:

$$
\begin{gathered}
\left(y_{1}, y_{2}\right)=y \mapsto U y=\left(U_{1} y, U_{2} y\right), \\
U_{k} y(t):=\int_{a}^{b} H_{k}(t, s) g_{k}\left(s, y_{1}(s), y_{2}(s)\right) d s, \text { for } k \in\{1,2\} .
\end{gathered}
$$

We have

$$
\begin{aligned}
\left|T_{k} x(t)-U_{k} x(t)\right| \leq & \int_{a}^{b} G_{k}(t, s) \mid f_{k}\left(s, x_{1}(s), x_{2}(s)\right) d s \\
& +\int_{a}^{b} H_{k}(t, s)\left|g_{k}\left(s, x_{1}(s), x_{2}(s)\right)\right| d s \\
\leq & \eta_{k} \int_{a}^{b} G_{k}(t, s) d s+\tau_{k} \int_{a}^{b} H_{k}(t, s) d s \\
\leq & \eta_{k}+\tau_{k}, \forall t \in[a, b], \text { for } k \in\{1,2\} \\
& \Rightarrow\left|T_{k} x-U_{k} x\right|_{\infty} \leq \eta_{k}+\tau_{k} \\
& \Rightarrow\|T x-U x\| \leq \eta+\tau, \forall x \in Z .
\end{aligned}
$$

The conditions of Theorem 6 are satisfied, so estimation (3.7) is proved.

Remark 1. A similar approach can be achieved for a system of Volterra type integral equations using, instead of the supremum norm, the Bielecki type norm approach.

\section{REFERENCES}

[1] W. A. Kirk, P. S. Srinivasan, and P. Veeramani, "Fixed points for mappings satisfying cyclical contractive conditions." Fixed Point Theory, vol. 4, no. 1, pp. 79-89, 2003.

[2] A. I. Perov and A. V. Kibenko, "About a general method for studying boundary value problems." Izv. Akad. Nauk SSSR, vol. 30, pp. 249-264, 1966.

[3] M. Păcurar and I. A. Rus, "Fixed point theory for cyclic $\varphi$-contractions." Nonlinear Analysis, vol. 72, pp. 1181-1187, 2010.

[4] I. A. Rus, Generalized Contractions and Applications. Cluj-Napoca: Cluj University Press, 2001.

[5] I. A. Rus, A. Petruşel, and G. Petruşel, Fixed Point Theory. Cluj-Napoca: Cluj University Press, 2001. 
Author's address

\section{Adrian Magdaş}

Babeş-Bolyai University, Faculty of Mathematics and Computer Science, Kogălniceanu Street, No. 1, 400084 Cluj-Napoca, Romania

E-mail address: amagdas@yahoo.com 